\title{
Chest compression effectiveness with vs without feedback
}

\author{
BinGe Yang ${ }^{1}$, Matthew J Douma ${ }^{2,3}$, Chris Picard ${ }^{4}$
}

${ }^{1}$ Faculty of Nursing, University of Alberta

${ }^{2}$ Royal Alexandra Hospital Emergency, Department of Critical Care Medicine, University of Alberta

${ }^{3}$ School of Nursing, University College of Dublin, Midwifery and Health Systems

${ }^{4}$ Misericordia Hospital Emergency, Edmonton, Alberta

\begin{abstract}
The objective of this experiment is to assess clinician perceived versus actual compression quality, and to evaluate the impact of using feedback from the Laerdal CPRMeter2 on compression quality. In our setup, we have a total of eighty four participants (43 from the Royal Alex and 41 from the Misericordia hospital). We monitored CPR quality based on the guidelines by Heart and Stroke, which breaks down chest compression effectiveness into three areasRelease, Depth and Rate. Proper Guidelines: Compress the chest at least 5cm (2inches); Compress at a rate of 100 to 120 beats per minute; Allow the chest to recoil completely after each compression. Clinical Setup: A convenience sample of participants performed two minutes of uninterrupted chest compressions on a Laerdal Resusci Anne with a CPRmeter2 on top without feedback, followed by a two minute rest period to fill out a Q.I tracking form. They later repeated the two minutes of chest compressions with the display of the CPRmeter uncovered, with the feedback visible. The chest compression metrics from the trials were compared using a data tracking form. From the data collected, enough evidence shows that the CPRmeter2 is able to improve release, rate and overall CPR quality. After filling out the survey, all of the nurses agree that the device is very useful in giving feedback and should be used in future CPR classes. Furthermore, data collected from the Q.I tracking forms indicates that nurses and other health clinicians are inadequate in predicting their own CPR abilities. Often times, nurses would either overpredict or underpredict their scores on the CPR meter.From the data gathered, the CPRmeter2 is going to be used for training in future CPR classes. Just recently, the device has been incorporated into code calls in the emergency department at the Misericordia. A T-test was done on the findings from the experiment to test if the means of two sets of data are significantly different from each other. Based on our findings, the t-test values for rate, release, and overall quality are statistically significant, meaning that the null hypothesis is rejected.
\end{abstract}

Key words:

CPR, chest compressions, cardiac arrest

Cite as: Yang B., Douma M., Picard C. 2019. Chest compression effectiveness with vs without feedback. Alberta Academic Review, Vol 2 (2) 83-84, WISEST Special Issue (non peer-reviewed), DOI 10.29173/aar77.

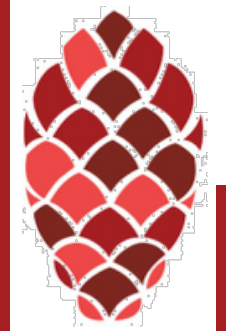


Yang et al., 2019

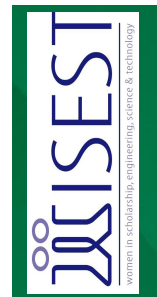

$\checkmark \frac{\widetilde{0}}{0}$

(3) 元

00 宛

(6) के के

(1) 0 용

5 융 즁

은 은

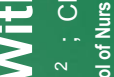

$\rightarrow \sum_{0}$

동

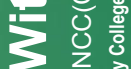

品

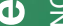

要

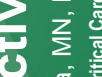

ब잉

ii

등

은 $\frac{1}{2}$

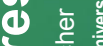

능

응

○ 항

के के

d

능

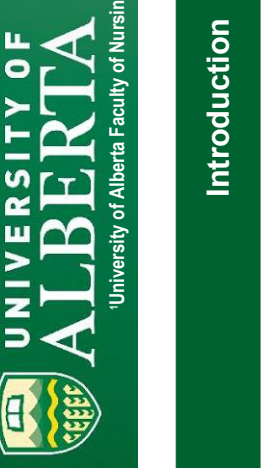

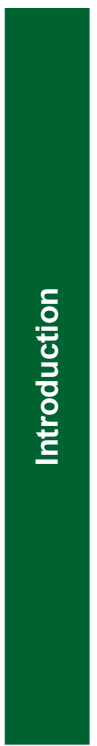

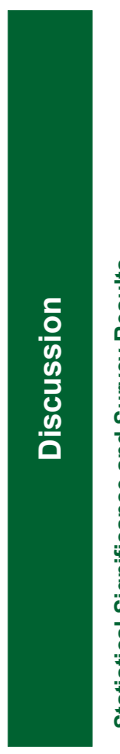
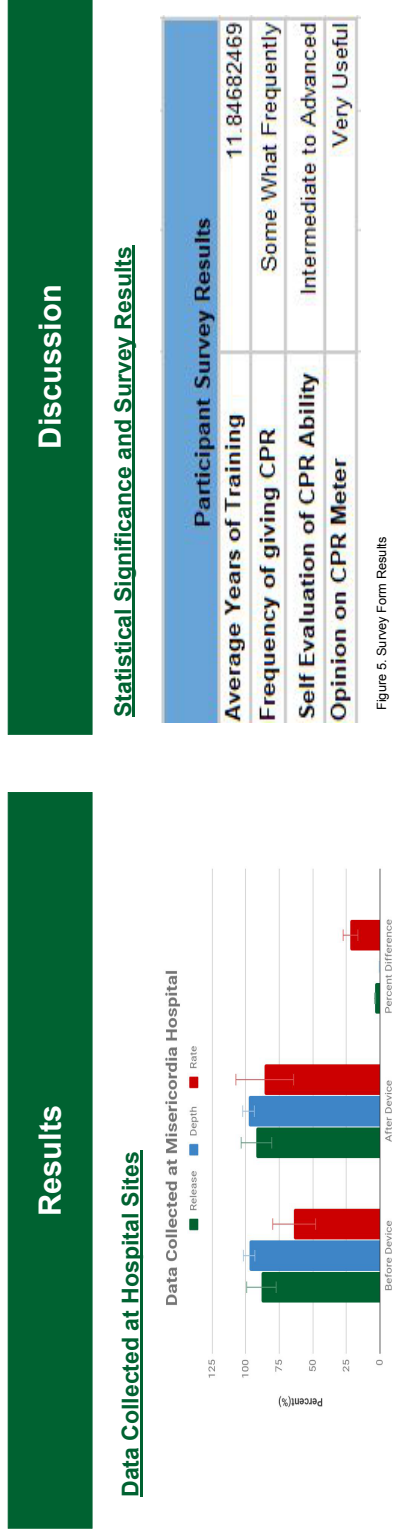
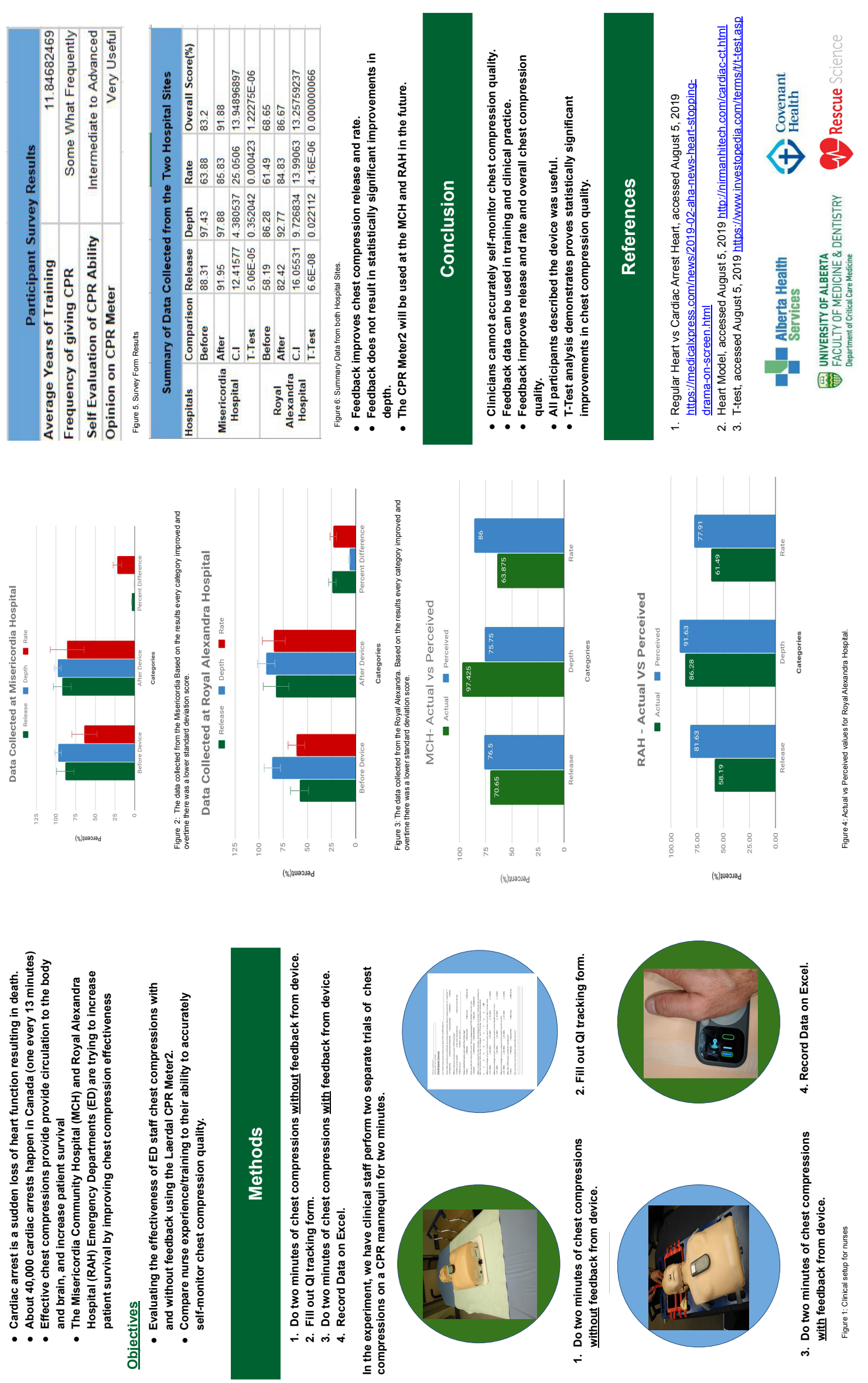

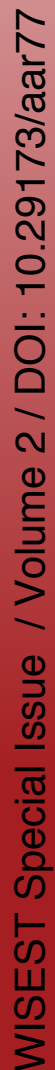
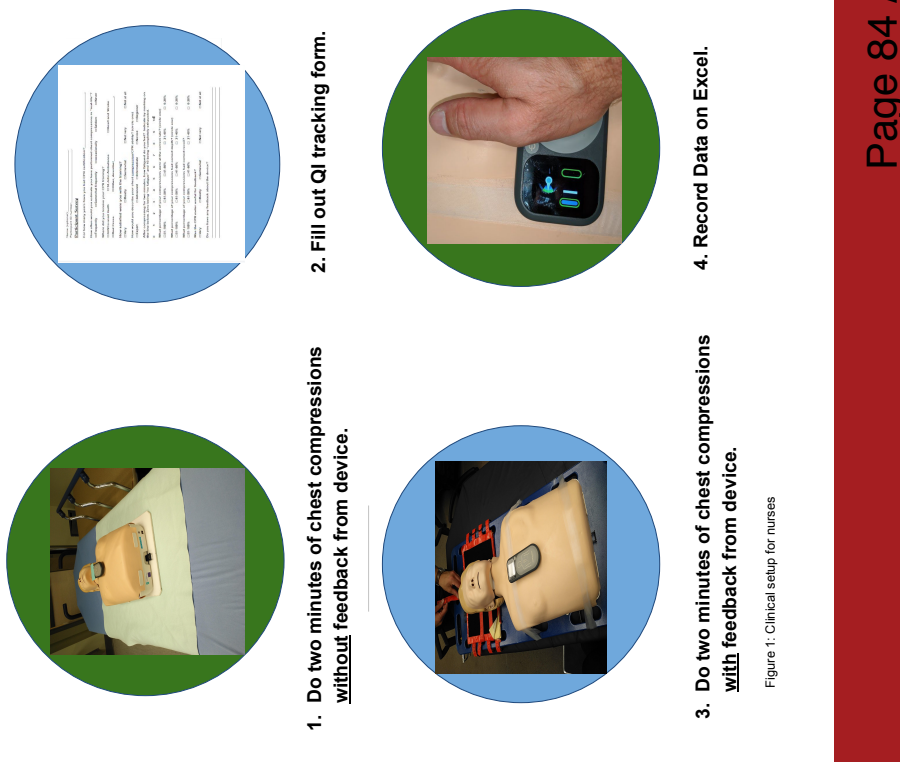